\title{
Apreciación de la guía de práctica clínica peruana sobre esquizofrenia respecto al programa de intervención temprana en psicosis
}

\author{
Appreciations about peruvian clinical guidelines in Schizophrenia in psychosis early intervention \\ program \\ Rubén Valle $1,2,3, a$ \\ Centro de Investigación en Epidemiología Clínica y Medicina Basada en Evidencias, Facultad de Medicina Humana, Universidad de San Martín de Porres. Lima, Perú. \\ 2 Facultad de Medicina, Universidad Nacional Mayor de San Marcos. Lima, Perú. \\ ${ }^{3}$ DEIDAE de Adultos y Adultos Mayores, Instituto Nacional de Salud Mental "Honorio Delgado-Hideyo Noguchi". \\ a Médico psiquiatra, epidemiólogo. ORCID: https://orcid.org/0000-0003-0811-200X
}

An Fac med. 2020;81(1):125-6. / https://doi.org/10.15381/anales.v81i1.17056

Correspondencia:

Rubén Eliseo Valle Rivadeneyra

ruben_vr12@hotmail.com

Recibido: 19 de noviembre 2019

Aprobado: 16 de diciembre 2019

Publicación en línea: 31 de marzo 2020

Fuentes de financiamiento:

Autofinanciado

Conflictos de intereses: El autor declara no tener conflictos de inetrés. Asimismo, los comentarios y opiniones mencionados en el presente artículo son de su autoría y no representan necesariamente la posición de las instituciones donde labora.

Citar como: Valle R. Apreciación de la guía de práctica clínica peruana sobre esquizofrenia respecto al programa de intervención temprana en psicosis. An Fac med. 2020;81(1):125-6. DOI: https://doi. org/10.15381/anales.v81i1.17056

\section{Sr. Editor,}

En el último número de su revista se ha publicado la guía de práctica clínica "El abordaje temprano y tratamiento mixto en el manejo de la esquizofrenia" (1). Este documento busca dar respuesta a cuatro preguntas clínicas referentes al manejo de este trastorno. Las respuestas a estas interrogantes se realizan en base a una adaptación de la guía "Psychosis and schizophrenia in adults: treatment and management" desarrollado por National Institute for Health and Care Excellence (NICE) ${ }^{(2)}$. Una de las interrogantes de la guía peruana fue "¿cuál es el beneficio de la intervención temprana en el primer episodio psicótico?". Es sobre el manejo del primer episodio psicótico que quisiera hacer algunas precisiones.

La guía NICE recomienda que las personas con un primer episodio de psicosis participen de los "Early intervention psychosis services" (2). Esta intervención se ha traducido al español, y aplicado en el campo clínico e investigación de los trastornos psicóticos como "programa de intervención temprana en psicosis" ${ }^{3,4,5)}$. En la guía peruana esta intervención ha recibido los nombres de "intervención temprana", "abordaje temprano" o "intervención terapéutica temprana" (1). Los distintos nombres no solo crean inconsistencias en la denominación de la intervención en la guía, sino que ninguno de ellos corresponde con el nombre de la intervención en español. Es importante aclarar este punto ya que "intervención temprana" per se en el campo de la psicosis solo hace referencia a uno de los múltiples componentes del programa ${ }^{(6,7)}$; y además, porque este mismo nombre es usado en otras áreas de la medicina como la pediatría donde hace referencia al conjunto de estrategias que se brindan a niños que presentan retraso en el desarrollo o discapacidad ${ }^{(8)}$.

Por otro lado, la guía menciona que "la intervención temprana en los servicios de psicosis deben procurar proporcionar la mayor gama de opciones farmacológicas, psicosociales, ocupacionales y educativas" ${ }^{(1)}$. Un programa de intervención temprana de psicosis, al igual que cualquier otro programa en salud, tiene componentes establecidos que se deben brindar de forma protocolizada ${ }^{(4,9)}$. Diversos estudios han identificado los componentes esenciales de los programas de intervención temprana en psicosis ${ }^{(6,7)}$, los cuales deben ser seguidos con una alta fidelidad si se quiere obtener los resultados esperados ${ }^{(10)}$. En ese sentido, es importante que los participantes reciban todos los componentes del programa que han mostrado eficacia y no solo aquellos que se encuentren disponibles ${ }^{(10)}$. Lo mencionado en la guía peruana supone que las estrategias del programa se pueden aplicar de forma parcial, lo cual no resulta apropiado ya que de esta forma no se obtendrían los resultados deseados ${ }^{(11)}$.

Un programa o servicio de intervención temprana en psicosis no se ha implementado en el Perú a pesar de la evidencia de su eficacia. Por ello, los pacientes que debutan con este cuadro reciben los mismos cuidados de salud que aquellos en estadios establecidos del trastorno. La presente guía sustenta la implementación de este programa, y es un llamado para que las autoridades de salud mental implementen de forma gradual, sistematizada y protocolizada esta intervención. 


\section{REFERENCIAS BIBLIOGRÁFICAS}

1. Salcedo LAV, Arévalo ET, Marín RÁV, Valenzuela CAS, Cruz MS de la, Clavijo GC, et al. El abordaje temprano y tratamiento mixto en el manejo de la esquizofrenia. An Fac Med. 2019;80(3):389-96. DOI: https://10.15381/anales.803.16869

2. National Collaborating Centre for Mental Health Psychosis and schizophrenia in adults: treatment and management: updated edition 2014. London: National Institute for Health and Care Excellence; 2014

3. Arango C, Bernardo M, Bonet P, Cabrera A, CrespoFacorro B, Cuesta MJ, et al. Cuando la asistencia no sigue a la evidencia: el caso de la falta de programas de intervención temprana en psicosis en España. Rev Psiquiatr Salud Ment. 2017;10 (2):78-86. DOI: 10.1016/j.rpsm.2017.01.001

4. Valle R. Revisión de los programas de intervención temprana de psicosis: propuesta de implemen- tación en Perú. Rev Colomb Psiquiat. 2018. DOI: https://doi.org/10.1016/j.rcp.2018.11.001

5. Programa de intervención temprana en psicosis [Internet]. Instituto Psiquiátrico Dr. José Horwitz Barak. 2019 [citado el 18 noviembre del 2019]. Disponible en: https://www.proitp.cl/

6. Marshall M, Lockwood A, Lewis S, Fiander M. Essential elements of an early intervention service for psychosis: the opinions of expert clinicians. BMC Psychiatry. 2004;4:17. DOI: 10.1186/1471244X-4-17

7. Addington DE, McKenzie E, Norman R, Wang J, Bond GR. Essential evidence- based components of first-episode psychosis services. Psychiatr Serv Wash DC. 2013:64 (5):452-7. DOI: 10.1176/appi. ps.201200156

8. CDC. ¿Qué es la "intervención temprana"? [Internet]. Centers for Disease Control and Prevention. 2019 [citado el 18 de noviembre del 2019]. Dis- ponible en: https://www.cdc.gov/ncbddd/spanish/ actearly/parents/intervencionTemprana.html

9. Nordentoft M, Rasmussen JO, Melau M, Hjorthøj CR, Thorup AAE. How successful are first episode programs? A review of the evidence for specialized assertive early intervention. Curr Opin Psychiatry. 2014;27(3):167-72 DOI: 10.1097/ YCO.0000000000000052

10. Csillag C, Nordentoft M, Mizuno M, Jones PB, Killackey E, Taylor M, et al. Early intervention services in psychosis: from evidence to wide implementation. Early Interv Psychiatry. 2016;10(6):540-6. DOI: 10.1111/eip.12279

11. Drake RE, Goldman HH, Leff HS, Lehman AF, Dixon $L$, Mueser KT, et al. Implementing evidence-based practices in routine mental health service settings. Psychiatr Serv Wash DC. 2001;52(2):179-82. DOI: 10.1176/appi.ps.52.2.179 